\title{
Geometry of Orientation and Ocular Dominance Columns in Monkey Striate Cortex
}

\author{
Klaus Obermayer ${ }^{1,2, a}$ and Gary G. Blasdel ${ }^{3}$ \\ 'Beckman Institute, University of Illinois at Urbana-Champaign, Urbana, Illinois 61801, ${ }^{2}$ The Rockefeller University, New \\ York, New York 10021, and 'Harvard Medical School, Boston, Massachusetts 02115
}

\begin{abstract}
In addition to showing that ocular dominance is organized in slabs and that orientation preferences are organized in linear sequences likely to reflect slabs, Hubel and Wiesel (1974a) discussed the intriguing possibility that slabs of orientation might intersect slabs of ocular dominance at some consistent angle. Advances in optical imaging now make it possible to test this possibility directly. When maps of orientation are analyzed quantitatively, they appear to arise from a combination of at least two competing themes: one where orientation preferences change linearly along straight axes, remaining constant along perpendicular axes and forming iso-orientation slabs along the way, and one where orientation preferences change continuously along circular axes, remaining constant along radial axes and forming singularities at the centers of the spaces enclosed. When orientation patterns are compared with ocular dominance patterns from the same cortical regions, quantitative measures reveal (1) that singularities tend to lie at the centers of ocular dominance columns, (2) that linear zones (arising where orientation preferences change along straight axes) tend to lie at the edges of ocular dominance columns, and (3) that the short iso-orientation bands within each linear zone tend to intersect the borders of ocular dominance slabs at angles of approximately $90^{\circ}$.
\end{abstract}

[Key words: striate cortex, orientation columns, ocular dominance columns, optical imaging, primate]

Hubel and Wiesel $(1962,1968,1974 a, b, 1977)$ established that most cells in striate cortex are binocular, that they nevertheless respond better to one eye, and that they usually respond best to a particular orientation. Hubel and Wiesel also established that cells selective for a particular eye or orientation are grouped together in slabs that extend vertically between pia and white

\footnotetext{
Received Aug. 31, 1992; revised Feb. 16, 1993; accepted Feb. 18, 1993.

This work was supported by the National Eye Institute (EY05403), the Offict of Naval Research, the National Science Foundation, the Klingenstein Foundation, the McKnight Foundation, the Center for Concurrent Biological Computing funded by NIH (P41RRO5969), and the New England Regional Primate Research Center (P51RRO168-31). We thank Helge Ritter and Klaus Schulten for stimulating discussions. We also thank Francis Crick, David Hubel, Marge Livingstone, Rick Born, Steve Macknik, and Gina Quinn for useful comments on the manuscript, and Sean Sommers and Mike Lafratta, who developed much of the specialized equipment. Susan Cowdery and Gina Quinn both provided excellent technical assistance.

Correspondence should be addressed to Gary G. Blasdel, Department of Neurobiology, Harvard Medical School, 220 Longwood Avenue, Boston, MA 02115. aPresent address: The Salk Institute, 10010 North Torrey Pines Road, La Jolla CA 92037.

Copyright $(C) 1993$ Society for Neuroscience $0270-6474 / 93 / 134114-16 \$ 05.00 / 0$
}

matter and that project laterally for some distance. In the case of ocular dominance, these slabs are $0.25-0.5 \mathrm{~mm}$ wide and several millimeters to several centimeters long. Subsequent work then established that cells dominated by a particular eye are grouped together in "ocular dominance" slabs, 0.2-0.4 mm wide, that project radially between pia and white matter and that can extend laterally for centimeters (Hubel and Wiesel, 1972; LeVay et al., 1975, 1980). Subsequent work also suggested that orientation preferences change linearly for lateral displacements through cortex, making it likely that these are organized in parallel slabs as well (Hubel and Wiesel, 1974a). In considering the evidence for slab-shaped orientation "columns," Hubel and Wiesel (1974a) considered the possibility that slabs of orientation and ocular dominance might intersect at some consistent angle.

Studies with the metabolic marker ${ }^{14} \mathrm{C}$-2-deoxyglucose (2DG) offered some hope of addressing these issues initially. By injecting this marker into animals stimulated with contours at a single orientation, Hubel et al. (1978) tried to determine the patterns of cells most activated by this orientation. What they observed were narrow bands of heightened activity in tangentially cut sections of cortex. Since these bands had the lengths, widths, and separations expected for "orientation columns," on the basis of single unit-recordings, they werc interpreted as orientation slabs cut in cross section, and since these presumed "orientation columns" intersected the borders of ocular dominance columns at all angles, their trajectories appeared to rule out any possibility of a consistent angle of intersection.

The interpretation of these bands as slabs of iso-orientation rested on several assumptions, however, one of which was that all cells in the upper layers are selective for orientation. As Horton and Hubel (1980), and later Livingstone and Hubel (1982, 1984), subsequently found, cells located in regions characterized by higher levels of cytochrome oxidase activity (WongRiley, 1979) contradict this assumption directly. Since these cells respond well to all orientations (Livingstone and Hubel, 1982, 1984), they always label (Horton and Hubel, 1980), irrespective of the orientation used to drive uptake. They also exhibit higher metabolic rates, consistent with their elevated levels of cytochrome oxidase, that cause them to takc up morc 2DG label than their neighbors, even at rest (Humphrey and Hendrickson, 1983).

The initial interpretation of this complication was that the nonselective labeling of blobs simply appears superimposed on the selective labeling of one set of iso-orientation slabs. However, for that to be the case, some blobs should lie between the selectively labeled iso-orientation bands, something that never is observed (Horton and Hubel, 1980). Since blobs always are 
embedded in 2DG-labeled iso-orientation bands, regardless of the orientation used to drive uptake, the only reasonable conclusion is that actual iso-orientation slabs are quite short, and that the only reason they appear longer, in 2DG material, is because the shorter slabs are strung together by blobs.

Another assumption, implicit in the interpretation of $2 \mathrm{DG}$ bands as regions preferring the orientation used to drive uptake, is that orientation preferences change linearly everywhere. Since this is not the case, and linearity is disrupted at regular intervals by breaks and reversals (Hubel and Wiesel, 1974a; Blasdel and Salama, 1986) that can also lead to spurious bands of label, there is a possibility that even the bands of 2DG label between cytochrome oxidase blobs cannot be trusted to reflect preferences for the orientation used to drive uptake (Blasdel, 1992a). The possibility of a simple geometric relation between slabs of ocular dominance and orientation therefore still exists.

Techniques of optical imaging (Blasdel and Salama, 1986; Blasdel, 1989a,b, 1992a,b; Frostig et al., 1990; Ts'o et al., 1990) now make it possible to address these issues directly by providing high-resolution maps of orientation preference and ocular dominance from the same cortical regions, in vivo. The maps of orientation preference revealed by this approach indicate that cells preferring similar orientations are indeed organized as slabs, in many regions of cortex, and they also reveal that these slabs are shorter than expected, extending laterally no farther than $0.5-1.0 \mathrm{~mm}$ (Blasdel, 1992a,b). Because linear sequences of iso-orientation slabs also extend laterally no farther than $0.5-1.0 \mathrm{~mm}$, these linear organizations are confined to twodimensional patches of similar dimensions, which are particularly abundant between the centers of adjacent ocular dominance columns.

The fact that short slabs of iso-orientation intersect the borders of ocular dominance columns at steep angles has been described briefly in previous publications (Blasdel, 1992b; Obermayer et al., 1992a,b) and confirmed recently by other investigators (Bartfeld and Grinvald, 1992). In this study we pursue this analysis further by applying quantitative measures at global and local scales. From our results we conclude (1) that orientation preferences are organized laterally by at least $t w o$ competing themes, generating linear as well as nonlinear compartments in complementary cortical regions; (2) that orientation preferences repeat at slightly longer intervals in directions perpendicular to the ocular dominance columns than in directions parallel; and (3) that within the linear zones, short slabs of iso-orientation may be discerned that intersect the borders of ocular dominance columns at steep, essentially perpendicular, angles.

\section{Materials and Methods}

\section{Optical imaging}

The techniques used to derive differential images of orientation and ocular dominance have been described previously in great detail (Blasdel and Salama, 1986; Blasdel, 1989a,b, 1992a,b). Briefly, each animal is anesthetized and surgically implanted with a stainless steel chamber that allows a portion of striate cortex to be stained with a voltagesensitive dye (NK2367) and stabilized against cortical pulsation. Reflectance changes of the dye-stained cortex are then visualized with a video microscope having a low power of magnification and a high numerical aperture, with a high dynamic range.

Each animal was paralyzed and refracted so that its eyes focused on a video monitor, $1.4-2.0 \mathrm{~m}$ away. The corneas were protected with hard, gas-permeable contact lenses. In order to align receptive fields from the two eyes with the center of the video screen, single units were recorded from the exposed cortex. Differential images of ocular dominance and orientation were then obtained according to established procedures (Blasdel and Salama, 1986; Blasdel, 1989a,b, 1992a,b). In all cases, visual stimuli consisted of four supcrimposed, squarc-wave gratings with nonharmonic spatial frequencies and $80 \%$ contrast, moving back and forth at 1.5 degrees/sec on the screen of the video monitor.

In order to obtain images of the ocular dominance bands, each eye was stimulated alternately with contours at one of four orientations. Video images averaged during stimulation of the left eye (four to six presentations for $1.5-3.0 \mathrm{sec}$, at intervals of 10-15 sec) were subtracted from images averaged during stimulation of the right eye, producing a final difference image that was then processed as described below, and normalized. Differential images of ocular dominance obtained at four equally spaced orientations, that is, at intervals of $45^{\circ}$, were then averaged together to produce the final image.

In order to record orientation preference and selectivity, the eyes were stimulated binocularly, with 4-12 different pairs of orthogonal contours presented four to six times each, in pscudorandom order. Differential images were obtained for each pair by subtracting the averaged image of cortex responding to contours at one orientation from averaged images of it responding to contours at the orthogonal orientation. After postprocessing (see below), all frames were converted to vector fields that were then added to yield two-dimensional maps of orientation preference and selectivity.

\section{Postprocessing}

Since the video images were obtained at an initial resolution of $512 \times$ 480 , each pixel corresponds to a horizontal distance of $8.6 \mu \mathrm{m}$ in the high-power images $(4.4 \mathrm{~mm} \times 3.3 \mathrm{~mm})$ and $15.6 \mu \mathrm{m}$ in the low-power images $(8 \mathrm{~mm} \times 6 \mathrm{~mm})$. Note that the horizontal spacing of video camera pixels differs from vertical. Pixels were then grouped into $4 \times$ 4 squares, averaged, and stored on disk at a resolution of $128 \times 120$. In order to remove unwanted noise components at very low spatial frequencies, the latter were isolated by convolving with a Gaussian kernel of half-width $\sigma=1.5 \mathrm{~mm}$, and subtracted from the original image, an approach that preserves the relative amplitudes of signals with spatial periods between $200 \mu \mathrm{m}(400 \mu \mathrm{m}$ at low power) and $2.0 \mathrm{~mm}$. Note, in Figure 1, that only the central portion of the images $(4.1 \mathrm{~mm} \times 3.0$ $\mathrm{mm}$ and $7.5 \mathrm{~mm} \times 5.6 \mathrm{~mm}$, respectively) are shown.

\section{Analysis}

\section{Notation}

In the following we will denote the position of a pixel within an image by $r$. The values of orientation preference, orientation selectivity, and ocular dominance at a pixel location $\mathbf{r}$ are abbreviated by $\Theta_{\mathrm{r}}, q_{\mathrm{r}}$, and $z_{\mathrm{r}}$, respectively. Positive and negative values of $z_{\mathrm{x}}$ indicate preference for the ipsi- and contralateral eye.

\section{Fourier transforms}

Fourier spectra of the orientation and ocular dominance maps are obtained by applying a two-dimensional fast Fourier transform (Press et al., 1988) to the final images after they have been compressed to a size of $512 \times 390$ pixels to correct for the different spacing of pixels along the vertical and horizontal axes of the video camera. All images are embedded in an array of size $512 \times 512$ and the values of orientation selectivity and ocular dominance were set to zero for the additional pixels. For the subsequent analyses only square windows were used. Power spectra estimations for orientation and ocular dominance maps have also been performed using Parzen windows, giving rise to similar results.

In the case of orientation preference and selectivity, the complex function $f_{\mathrm{r}}=q_{\mathrm{r}}\left(\cos \left(2 \Theta_{\mathrm{r}}\right)+\sin \left(2 \Theta_{\mathrm{r}}\right)\right)$ and, in the case of ocular dominance, the function $f_{\mathrm{r}}=z_{\mathrm{r}}$, are transformed with respect to the cortical location r. The squared values $\left|\hat{f}_{\mathbf{k}}\right|^{2}$,

$$
\hat{f}_{\mathbf{k}}=\int_{\mathbf{r}} f_{\mathrm{r}} \exp (-i \mathbf{k r}) d \mathbf{r},
$$

of the corresponding Fourier amplitudes are then plotted as a function of the wave vector $\mathbf{k}=\left(\mathbf{k}_{1}, \mathbf{k}_{2}\right)$. The resolution in spatial frequency is $0.23 / \mathrm{mm}$ for animals NM1 and NM2, and $0.13 / \mathrm{mm}$ for animals NM3, NM4, FS1-1, and FS1-2. 


\section{Estimation of the characteristic wavelengths of orientation and} ocular dominance columns

Average wavelength of the orientation map. Power spectra of the orientation map are approximated by averaging the corresponding Fourier amplitudes $\left|\hat{V}_{\mathbf{k}}\right|^{2}$ over all directions of the wave vector $\mathbf{k}$. The average power as a function of spatial frequency typically shows one pronounced peak (Obermayer et al., 1991, 1992a,b). The wavelength $\lambda^{\text {op }}$, which corresponds to the peak, is used to define the average wavelength of the orientation column system. Another possible definition of the average wavelength involves calculating the power density as a function of spatial frequency. The power density can be approximated by integrating the Fourier spectra along circles around the origin. The power density as a function of spatial frequency also exhibits one peak. The inverse $\hat{\lambda}^{\mathrm{OP}}$ of peak frequency is typically $40 \mu \mathrm{m}$ smaller than $\lambda^{\mathrm{OP}}$ (see Table 1 ).

Average wavelength of the ocular dominance map. The Fourier spectra of the ocular dominance maps typically show two noisy peaks that are located on opposite sides of the origin. The inverse $\lambda^{\circ D}$ of the spatial frequency that corresponds to the distance of the peaks from the origin is used to define the average wavelength of the ocular dominance column system.

Wavelength of the orientation map parallel and perpendicular to the ocular dominance bands. The squared Fourier amplitudes $\left|\hat{f_{k}}\right|^{2}$ of the orientation map are averaged over all wave vectors $\mathbf{k}$, for every length $|\mathbf{k}|$, whose directions deviate less than $15^{\circ}$ from the axes perpendicular and parallel to the ocular dominance bands, respectively. These axes can be determined from the Fourier spectra of ocular dominance. They are given by the axes connecting both peaks and by the orthogonal axes, respectively.

Diagrams of the average power as a function of spatial frequency typically exhibit one noisy peak. The wavelengths $\lambda_{1}^{O P}$ and $\lambda_{1}^{\mathrm{OP}}$ corresponding to these peaks are used to define the average wavelengths of the orientation map parallel and perpendicular to the ocular dominance bands, respectively.

\section{Parallelism}

For a quantitative analysis of orientation preference and ocular dominance it is useful to have a measure for the degree to which regions of similar orientation preference or similar ocular dominance are organized locally into parallel slabs. We call this a measure of parallelism and we abbreviate it by $P^{O P}$ and $P O D$, respectively.

The gradient of orientation preference at every location $\mathbf{r}$ is approximately given by

$$
\begin{aligned}
& \left(\nabla_{\mathbf{r}} \Theta_{\mathbf{r}}\right)_{1}=(1 / 2 \Delta) \Pi\left(\Theta_{r_{1}+1, r_{2}}-\Theta_{r_{1}-1, r_{2}}\right), \\
& \left(\nabla_{\mathbf{r}} \Theta_{\mathrm{r}}\right)_{2}=(1 / 2 \Delta) \Pi\left(\Theta_{r_{1}, r_{2}+1}-\Theta_{r_{1}, r_{2}-1}\right),
\end{aligned}
$$

where $\Delta$ is the size of one pixel in the image and where the function $\Pi$,

$$
\Pi(x)= \begin{cases}x, & \text { if }|x|<\frac{1}{2} \pi, \\ x-\pi, & \text { if } x>\frac{1}{2} \pi, \\ x+\pi, & \text { if } x<-\frac{1}{2} \pi,\end{cases}
$$

takes into account that orientation preference is a cyclic quantity. The gradient of orientation preference is therefore a two-dimensional vector whose length denotes the local rate of change and whose direction indicates the axis along which this rate is greatest. Note that the Equations 2 and 3 approximate the gradient only when the difference in orientation preference between neighboring pixels is small.

In order to derive a direct measure of "parallelism," it is useful to characterize the directions of iso-orientation lines with unit vectors whose angular components correspond to the angle between the isoorientation contour and a reference axis, multiplied by 2 . These vectors can be obtained readily from vectors indicating the gradient of orientation preference by normalizing their lengths, rotating them by $90^{\circ}$ (by adding $\pi / 2$ to their angular components), and multiplying the resulting angles by 2 . All unit vectors located in a circular region of radius $\sigma_{g}$ are then averaged, producing a vector $P^{O P}$, whose length $P^{O P}$ becomes a measure of parallelism. Values of 1.0 indicate regions where the isoorientation lines are perfectly parallel; values smaller than 1.0 indicate regions where the iso-orientation lines are less aligned, and values of 0.0 indicate singularities. This measure of parallelism has biological significance when $\sigma_{g}=100 \mu \mathrm{m}$, since this corresponds to the approximate radius of upper layer pyramidal dendrites.

The measure $P O D$ of "parallelism" for ocular dominance bands can be calculated in a similar way. The function $\Pi$ in Equation 2, however, has to be changed to

$$
\Pi(x)=x, \quad \text { for all } x .
$$

Throughout this article we will call a region in monkey striate cortex a linear zone, if $P^{\mathrm{OP}}$ exceeds a value of 0.6 for $\sigma_{g}=100 \mu \mathrm{m}$.

\section{Intersection angle between orientation and ocular dominance bands}

The average, local directions of iso-orientation and ocular dominance contours are given by the angular components of the vectors $\mathbf{P}^{\circ \mathrm{P}}$ and POD, divided by 2 . From these components it is possible to calculate the local intersection angle $\alpha$ between iso-orientation and ocular dominance contours by

$$
\alpha=\Pi\left(0.5 \cdot\left|\phi^{\mathrm{OP}}-\phi^{\mathrm{OD}}\right|\right)
$$

where $\phi^{\mathrm{OP}}$ and $\phi^{\mathrm{OD}}$ are the angular components of the vectors $P^{O P}$ and $P^{O D}$, and where the function $\Pi(x)$,

$$
\Pi(x)= \begin{cases}x, & \text { if } x<\frac{1}{2} \pi, \\ \pi-x, & \text { if } x>\frac{1}{2} \pi,\end{cases}
$$

takes into account that the intersection angle is a cyclic quantity.

Contours of equal orientation preference (or ocular dominance) are aligned in regions where the measures $P^{\mathrm{OP}}$ (Or $P O D$ ) are close to 1 . In these regions bands of similar orientation preference and ocular dominance exist, and their local intersection angle is given by $\alpha$.

\section{Results}

Maps of the vasculature, ocular dominance, and orientation

Figure 1 displays ocular dominance and orientation patterns from six separate regions in five animals (four Macaca nemestrina, one Macaca fascicularis). Maps of ocular dominance and orientation preference appear in the second and third columns, beside images of the vasculature in the first column with horizontal and vertical axes subtending $4.1 \mathrm{~mm}$ and $3.0 \mathrm{~mm}$ for $\mathrm{NM} 1$ and NM2 (top two rows), and $7.5 \mathrm{~mm}$ and $6.5 \mathrm{~mm}$ for the others. For NM3, NM4, and FS1-1, the lower border of each area was located $2-4 \mathrm{~mm}$ above the boundary of striate cortex (the $17 / 18$ border). For NM1, NM2, and FS1-2 the 17/ 18 border was located more than $10 \mathrm{~mm}$ below the bottom of each frame. A visual comparison between the vascular patterns and the maps of ocular dominance and orientation preference reveals a few artifacts generated by major blood vessels. These regions, which are indicated by hatching over the vascular images, were excluded from all quantitative analyses.

\section{Fourier spectra and characteristic wavelengths of the orientation and ocular dominance columns}

Global properties of repetitive patterns are extracted most easily by transforming them from the spatial domain into frequency

Figure 1. Differential images of orientation, ocular dominance, and the corresponding vascular patterns obtained from six different adult macaques. The horizontal axis in each frame corresponds to $4.1 \mathrm{~mm}$ for NM1 and NM2, and to $7.5 \mathrm{~mm}$ for NM3, NM4, FS1-1, and FS1-2. Left column, Vascular patterns recorded under white light. The hatched areas in the vascular patterns indicate regions where blood vessels give rise to artifacts in the differential images of orientation or ocular dominance. Right column, Orientation preference. Orientation preference is indicated by color, 


\section{NM1}
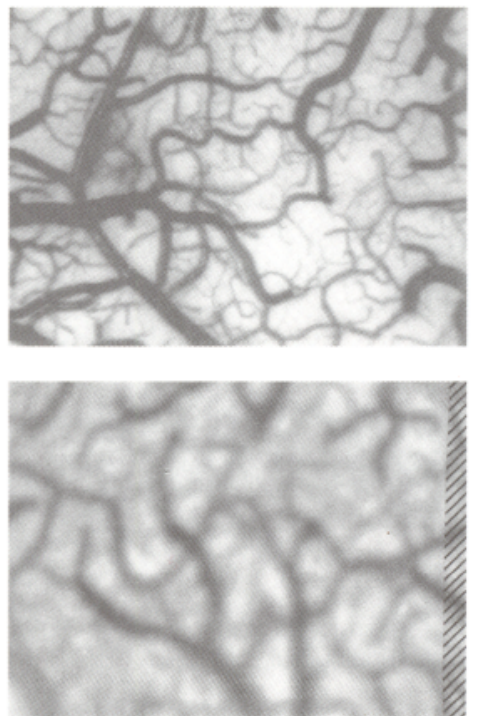

NM2

NM3
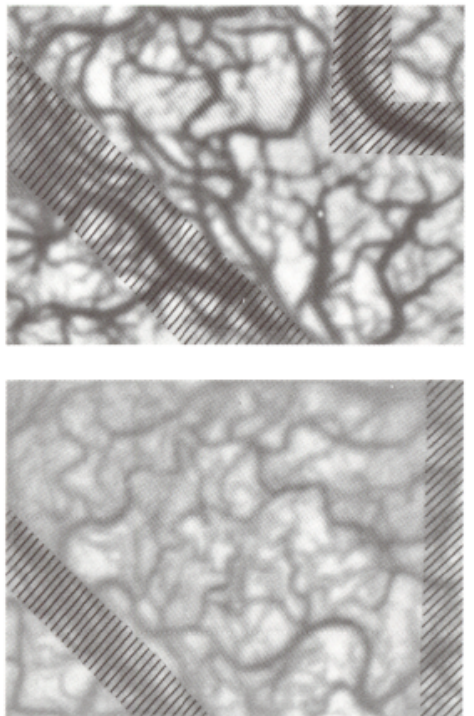

NM4

FS1-1
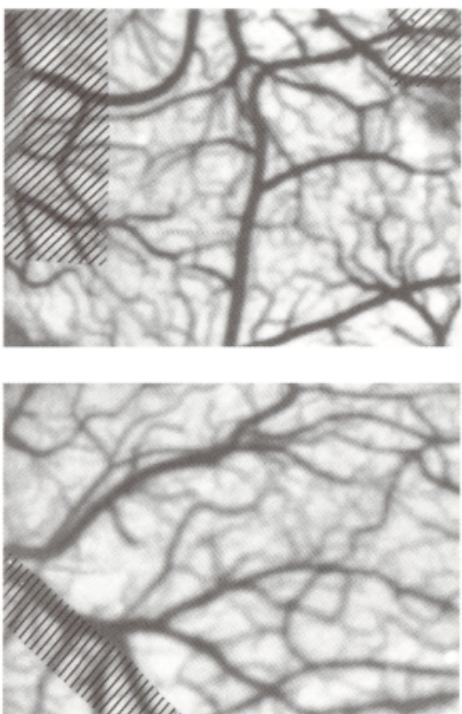

FS1-2
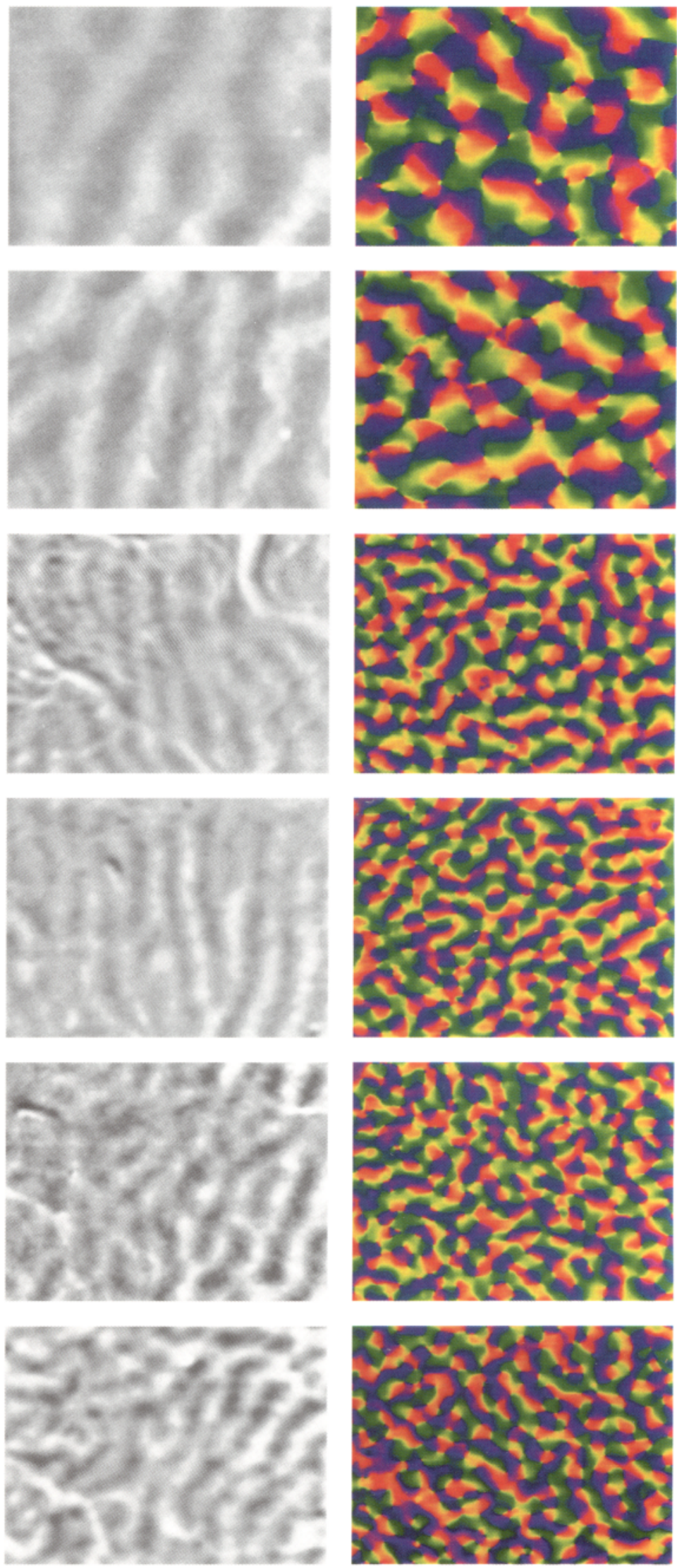

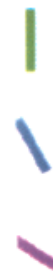

where the sequence red $\rightarrow$ yellow $\rightarrow$ green $\rightarrow$ blue $\rightarrow$ purple $\rightarrow$ red describes a complete cycle of preferred orientations from horizontal through right oblique, vertical, left oblique, and back to horizontal. Complementary colors indicate orthogonal orientations. Center column, Ocular dominance, coded by brightness, where dark areas indicate stronger response for stimulation of the right eye, bright areas indicate stronger response for stimulation of the left eye, and gray areas indicate equal response for stimulation of either eye. 


\begin{tabular}{|c|c|c|c|c|c|c|c|c|}
\hline Animal & $\lambda^{\mathrm{OP}}$ & $\hat{\lambda}^{\text {OP }}$ & $\lambda^{\mathrm{OD}}$ & $\lambda_{\perp}^{\mathrm{OP}}$ & $\lambda_{\|}^{O P}$ & $\lambda^{\mathrm{OP} / \lambda^{\mathrm{OD}}}$ & $\lambda_{\perp}^{\mathrm{OP} / \lambda^{\mathrm{OD}}}$ & $\lambda_{\|}^{\mathrm{OP} / \lambda^{\mathrm{OD}}}$ \\
\hline NM1 & 768 & 733 & 903 & 928 & 653 & 0.85 & 1.03 & 0.72 \\
\hline NM2 & 645 & 615 & 773 & 723 & 701 & 0.83 & 0.94 & 0.91 \\
\hline NM3 & 621 & 588 & 796 & 709 & 560 & 0.78 & 0.89 & 0.70 \\
\hline NM4 & 719 & 670 & 829 & 745 & 694 & 0.87 & 0.90 & 0.84 \\
\hline FS 1-1 & 676 & 623 & 829 & 776 & 596 & 0.82 & 0.94 & 0.72 \\
\hline FS 1-2 & 647 & 611 & 800 & 667 & 628 & 0.81 & 0.83 & 0.79 \\
\hline Average & 679 & 640 & 822 & 758 & 639 & 0.83 & 0.92 & 0.78 \\
\hline
\end{tabular}

The estimated error for the individual wavelengths is $\pm 50 \mu \mathrm{m}$.

space (see, e.g., Papoulis, 1984). When Fourier analysis is applied to images of ocular dominance, it generates Fourier spectra with two clusters of modes displaced in opposite directions from the origin, along the axis of repetition (Fig. 2, center column). Because the radial and tangential axes represent characteristic frequencies and directions, scatter along the radial axis reflects the variability in frequency, which is $1.22 \pm 0.05 / \mathrm{mm}$ on average (all error margins indicate standard error), while scatter in the perpendicular direction, along tangential axes, reflects variability in direction among the different ocular dominance bands.

Images of orientation preference (Fig. 2, left column), by contrast, give rise to spectra with nearly circular bands of modes, which indicate that orientation preferences repeat in every direction. The diameter of each ring reflects the spatial frequency with which orientation preferences repeat along any particular axis, with an average repetition of $1.47 \pm 0.07 / \mathrm{mm}$ on average, while the width of the ring along each axis corresponds to the range of frequencies represented. From the average widths of these circular bands it is possible to calculate a coherence length of $820 \pm 130 \mu \mathrm{m}$, which is the typical interval over which orientation values can be said to change periodically and linearly. The coherence length can also be calculated in the spatial domain, where an analysis of the corresponding correlation functions leads to the same result (Obcrmaycr ct al., 1991, 1992a,b).

When the Fourier spectra of orientation preferences in Figure 2 are examined closely it becomes apparent that all of them are elliptic. This is because the frequency of repetition varies with direction, creating major and minor axes. In Figure 2 (left two columns), the major axes, corresponding to the highest rates of repetition for orientation and ocular dominance, are indicated by solid black lines whose orientations can be compared. As one can verify easily for any sample, the major axes for orientation and ocular dominance intersect one another steeply, often at perpendicular angles. From Table 1 one can see that this is because orientation preferences repeat every $758 \pm 37 \mu \mathrm{m}$ along axes that are perpendicular to the major axis for ocular dominance while they repeat every $639 \pm 23 \mu \mathrm{m}$ along axes that are parallel. The maps of orientation preference therefore appear stretched perpendicular to the ocular dominance bands.

Further insight into the arrangement of orientation and ocular dominance can be gained from the right column of Figure 2, where the mean square amplitudes of all Fourier modes of oricntation preference have been added and plotted against the corresponding angles of intersection. If orientation bands tend to align with one another, as well as with a particular axis, then the power should be high along the axis of repetition and low along the axis of alignment. This is the result one sees in the third column of Figure 2, where the power fraction of orientation spectra is higher in directions running parallel to the ocular dominance bands (perpendicular to their wave vectors) than it is in directions running perpendicular. This suggests that isoorientation bands intersect ocular dominance bands at roughly perpendicular angles.

Geometrical relations between orientation and ocular dominance columns are difficult to discern from Fourier spectra alone, however. This is because trends are averaged over large areas, and local correlations, like the local angles of intersection, are lost in areas that are irregular. As one can verify easily in Figure 2, the cllipticity of the Fourier spectra of orientation preference is less pronounced for cases where the ocular dominance bands are less parallel. In order to provide strong evidence for consistent angles of intersection, therefore, one has to limit the scale of analysis to regions smaller than the coherence length, to regions where the orientation and ocular dominance bands are less variable.

\section{Local organization of orientation preference}

Because orientation preference changes continuously, in cycles, it cannot be represented by the light/dark values of a gray-tone image. As Blasdel and Salama (1986) pointed out initially, it is

Figure 2. Fourier analysis of the differential images of orientation preference and ocular dominance. Left column, Fourier spectra of the orientation map. Each mode corresponds to one pixel, and its gray values indicate its squared amplitude. All Fourier spectra are characterized by a slightly elliptic band of values. The solid lines indicate their major axis. Center column, Fourier spectra of the corresponding ocular dominance pattern. Images of ocular dominance give rise to Fourier spectra with two clusters displaced in opposite directions from the origin. The solid lines indicate the axes of repetition, which are identical with the axes connecting both clusters. Note, that the axes of fast repetition of orientation preferences and ocular dominance (solid lines) tend to intersect each other at steep angles. Right column, Power as a function of the direction of the wave vector for the orientation map. The mean squared amplitude of the Fourier modes was summed over all modes, whose wave vectors had similar angles with the axes of repetition of the ocular dominance bands. Modes that correspond to a spatial frequency higher than $10 / \mathrm{mm}$ and $5 / \mathrm{mm}$ for the high- and low-power images, respectively, and smaller than $0.4 / \mathrm{mm}$ were discarded. The diagrams show the fraction of the power in each of the angle intervals $-15^{\circ}-15^{\circ}, 15^{\circ}-45^{\circ}, 45^{\circ}-75^{\circ}, 75^{\circ}-105^{\circ}, 105^{\circ}-135^{\circ}$, and $135^{\circ}-165^{\circ}$. 
orientation preference

NM1

NM2

NM3

NM4

FS1-1

FS1-2
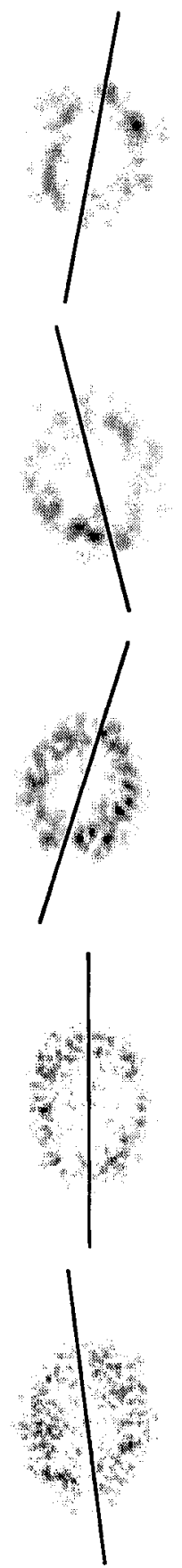
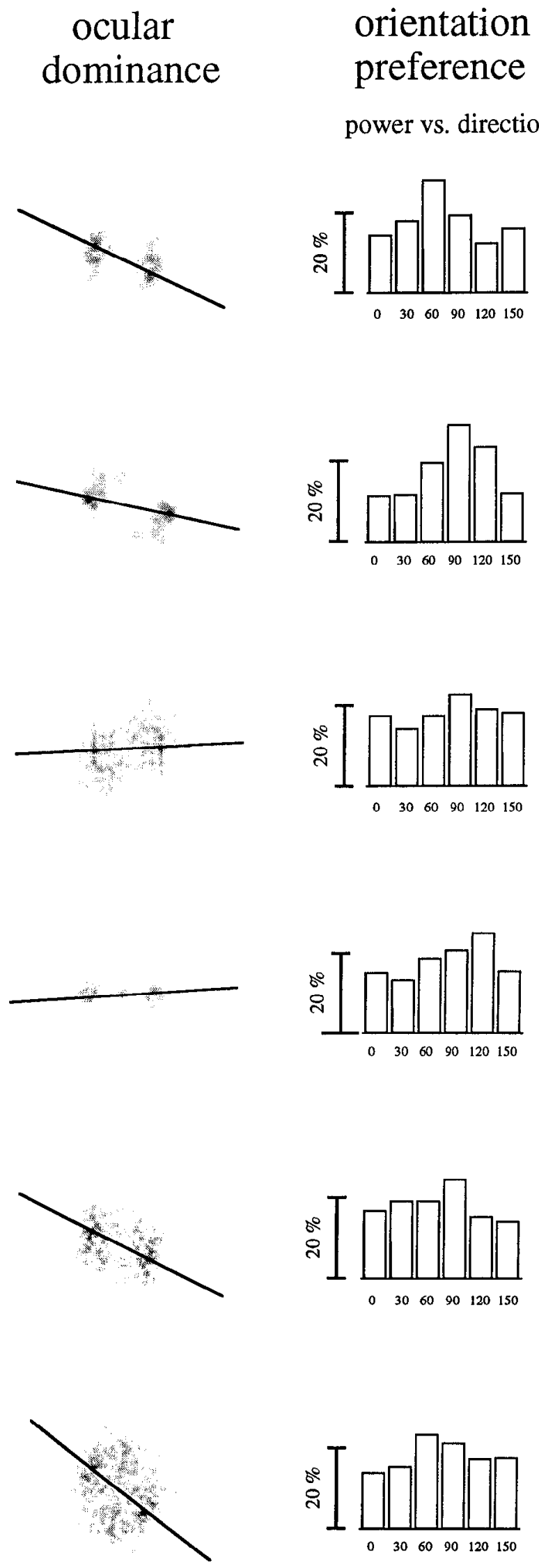
Table 2. Percentage of cortical area covered by linear zones and density of singularities

\begin{tabular}{lllll} 
& $\begin{array}{l}\text { Linear } \\
\text { zones } \\
\text { (\% of area) }\end{array}$ & $\begin{array}{l}\text { Density of } \\
+180^{\circ} \\
\text { singular- } \\
\text { ities }\left(\mathrm{mm}^{-2}\right)\end{array}$ & $\begin{array}{l}\text { Density of } \\
-180^{\circ} \\
\text { singular- } \\
\text { ities }\left(\mathrm{mm}^{-2}\right)\end{array}$ & $\begin{array}{l}\text { Total } \\
\text { density of } \\
\text { singular- } \\
\text { ities }\left(\mathrm{mm}^{-2}\right)\end{array}$ \\
\hline NM1 & 43 & 3.9 & 3.8 & 7.7 \\
NM2 & 35 & 4.7 & 4.7 & 9.4 \\
NM3 & 56 & 3.9 & 3.8 & 7.7 \\
NM4 & 58 & 3.6 & 3.2 & 6.8 \\
FS1-1 & 47 & 4.4 & 4.4 & 8.8 \\
FS1-2 & 50 & 4.2 & 4.0 & 8.2 \\
Average & 48 & 4.1 & 4.0 & 8.1
\end{tabular}

represented more easily in color, where rotations around color space can be used to represent rotations through $180^{\circ}$ continuously, and where complementary colors (e.g., red vs green, or blue vs yellow) can be used to represent the most-different relationships between orthogonal orientations (e.g., horizontal vs vertical, or left oblique vs right oblique; see Fig. 1). The use of color has limitations as well, however, which can lead to suggestions that preferences for some orientations outnumber preferences for others. As one can deduce from the contour plots in Figures $3 b, 4 b$, and 5 , where comparable intervals of orientation preference may be seen to cover approximately equal areas, this is probably not the case.

Figure 3, $a$ and $b$, depicts an enlarged section of the orientation map derived from animal FS1-2, with arrows to indicate typical elements, which are defined as follows.

Linear zones. These are the two-dimensional patch-like zones where orientation preferences change linearly along one axis while remaining constant along the other. They are $0.5-1.0 \mathrm{~mm}$ across, and give rise to the rainbow-like patterns apparent in Figure $3 a$. Additional information can be extracted from the contour plot of orientation preferences in Figure $3 b$, where isoorientation contours are drawn for orientation preferences in increments of $15^{\circ}$. The similar spacing of these contours in linear zones indicates that orientation preferences change linearly, as well as continuously, with distance in the perpendicular direction. A linear regression analysis for values of orientation preference, as a function of cortical distance along the black line in Figure $3 a$, for example, yields a correlation coefficient of 0.994 , with orientation preferences changing at an average rate of $32^{\circ}$ per $100 \mu \mathrm{m}$, or $180^{\circ}$ every $0.56 \mathrm{~mm}$.

Singularities. These are zero-dimensional, point-like discontinuities that arise when orientation preferences change continuously through $\pm 180^{\circ}$ along any closed path around them (Blasdel, 1992b; Obermayer et al., 1992a,b). Positive and negative singularities appear in equal numbers with an average density of $8.1 / \mathrm{mm}^{2}$ (see Table 2 ). In most cases, singularities with opposite signs (e.g., one positive and one negative) are connected by iso-orientation bands running in between. In other cases, however, singularities of similar sign may also be connected, in which case they give rise to saddle points, as described below.

Fractures. Fractures are one-dimensional discontinuities across which orientation preferences change rapidly (Blasdel and Salama, 1986; Blasdel, 1992b). They are known from tangential electrode recordings (Hubel and Wiesel, 1974a; Blasdel and Salama, 1986) and can easily be detected in the contour plots in Figures $3 b$ and 5 , where they give rise to closely spaced iso- orientation contours. Like bands of iso-orientation, fractures connect singularities of opposite sign.

Saddle points. These are small, two-dimensional patches, where orientation preference remains approximately constant. Outward movements from the middle of a saddle point tend to generate similar rotations in orientation preference for movements in opposite directions. Along one axis, orientation preference may rotate clockwise for movements in either direction from the middle, while similar movements along the orthogonal axis generate counterclockwise rotations. Saddle points are bounded by four singularities that are often, but not always, connected by fractures.

The small section displayed in Figure 3 already indicates that singularities, fractures, and saddle points are grouped together in more structured arrangements that stand in marked contrast to the smooth, continuous changes in orientation preference that characterize the linear zones. These groupings can be found by visual inspection in several places within the maps in Figure 1 (right column). For a more rigorous analysis, however, it is useful to consider measures that quantify the geometry of these patterns in small cortical regions as described in Materials and Methods.

The vectors obtained by taking the gradient of orientation preference indicate directions of change. When the angles of these vectors are doubled, directional components are lost and orthogonal axes of change become opposites. Consequently, the local linearity, which can be approximated by the parallelism of iso-orientation contours, can be extracted along with their axis of alignment by normalizing the doubled vectors, averaging them over small patches and dividing the angular components of the averaged vectors by 2 . The length $P^{\mathrm{OD}}$ of each resulting vector reflects the degree to which iso-orientation contours are aligned in every region; this may be taken as a measure of linearity. While values of 1.0 and values close to 1.0 indicate linear zones and parallelism, values closer to zero reflect increasing levels of disorder, with values of zero reflecting no alignment or linearity at all. In the vicinity of a singularity, for example, this measure of linearity is usually zero.

Figure $4 a$ shows the result of this analysis applied to NM1, where the darkest regions indicate the most linear zones, which exhibit the highest levels of alignment, and the bright areas represent nonlinear zones, characterized by singularities, fractures, and saddle points. This correspondence is readily apparent in Figure $4 b$, where regions expressing linearity $\left(P^{\mathrm{OP}}\right)$ greater than 0.6 are illustrated in gray and overlaid with iso-orientation contours. As one can see, the gray zones correspond to sets of evenly spaced parallel lines, while the white zones correspond to singularities (where many contours run together), fractures (where the contours run parallel, but much closer together), and saddle points (where the contour lines diverge). One should keep in mind, however, that the ratio of areas covered by linear and nonlinear zones also depends on the size of areas within which iso-orientation axes are averaged. If these were smaller than the 100 - $\mu \mathrm{m}$-radius zones used in these examples, the proportion of zones characterized as linear would increase.

\section{Angles of intersection between orientation and ocular dominance bands}

Figure $5 a$ shows iso-orientation contours superimposed on the borders of ocular dominance bands for NM1, where, from visual inspection, it is obvious that where parallel slabs of iso-orientation are defined they intersect the borders of ocular dominance 

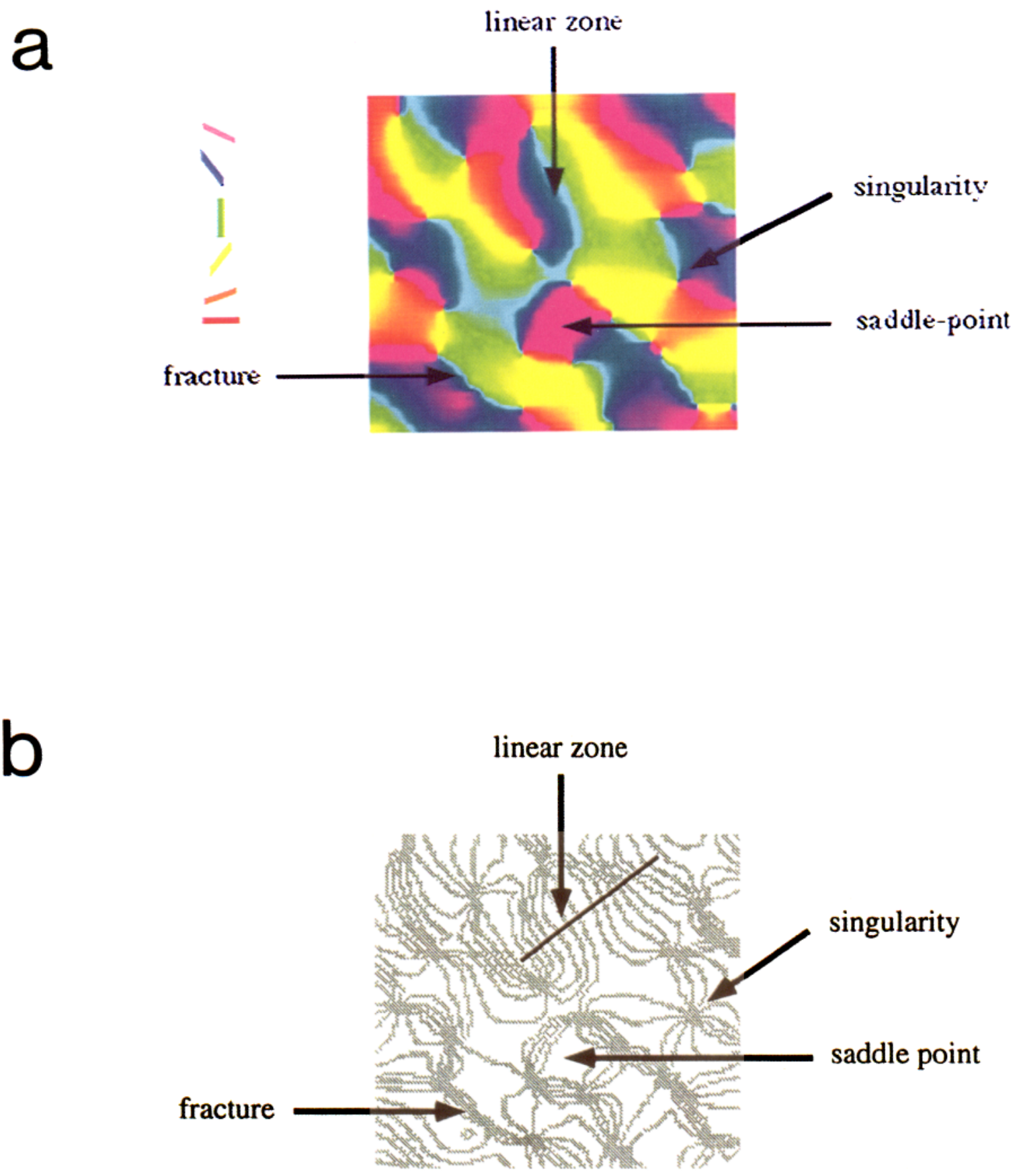

Figure 3. This figure shows an enlarged section of the image of orientation preference of animal FS1-2. $a$, Color-coded map of orientation preference. Arrows indicate linear zones, singularities, fractures, and saddle points. $b$, Contour plot of orientation preferences for the same area. Iso-orientation contours are drawn in intervals of $15^{\circ}$. The horizontal axis corresponds to $1.7 \mathrm{~mm}$.

slabs at approximately right angles. One highly ordered example appears in Figure $5 b$, which is enlarged from a portion of Figure $5 a$. As one can see form this example, singularities have a tendency to lie in the centers of ocular dominance bands, as reported previously (Blasdel, 1992b; Swindale, 1992). This tendency has two consequences: (1) singularities in adjacent ocular dominance columns tend to be connected by iso-orientation contours, and (2) singularities in the same ocular dominance column tend to be linked by saddle points.

Because iso-orientation contours tend to run between singularities in adjacent ocular dominance columns, most of them cross the borders between ocular columns at steep angles, mak- ing them orthogonal. With our measure of parallelism, $P^{\mathrm{OP}}$, it is possible to measure this orthogonality quantitatively. Figure $6 a$ shows a histogram of the intersection angles between isoorientation and ocular dominance slabs in regions characterized by $P^{O P}>0.6$, where it becomes clear that iso-orientation slabs are virtually perpendicular $\left( \pm 18^{\circ}\right)$ to ocular dominance slabs $46 \%$ of the time, and that regions where they are perpendicular $\left( \pm 18^{\circ}\right)$ are at least seven times as abundant as those where they are parallel $\left( \pm 18^{\circ}\right)$. These differences go away when ocular dominance values are randomized before calculating the orientation of ocular dominance bands (Fig. $6 b$ ), and they reemerge in the opposite direction when the ocular dominance pattern is rotated 


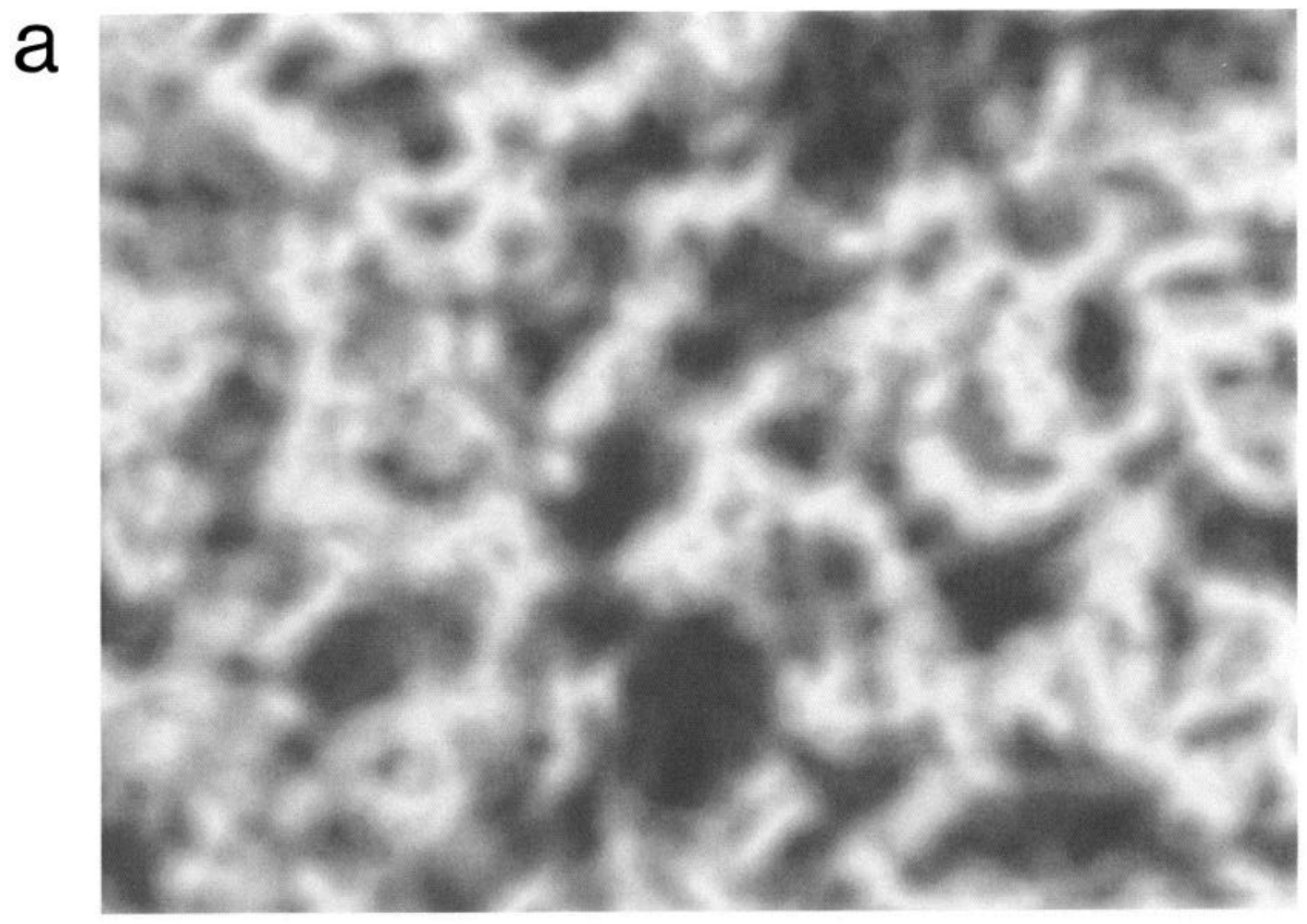

Figure 4. Quantitative analysis of shape and arrangement of regions with similar orientation preference. $a$, This image shows the measure $P O P$ of "parallelness" (see Materials and Methods) for the orientation map of animal NM1. Dark and bright areas indicate regions of high and low values of $P^{O P}$, respectively. $\sigma_{g}$ was 100 $\mu \mathrm{m}$. $b$, This image shows regions with $P^{O P}>0.6$ (gray) in overlay with a contour plot of orientation preferences. Neighboring iso-orientation lines are drawn in intervals of $11.25^{\circ}$.

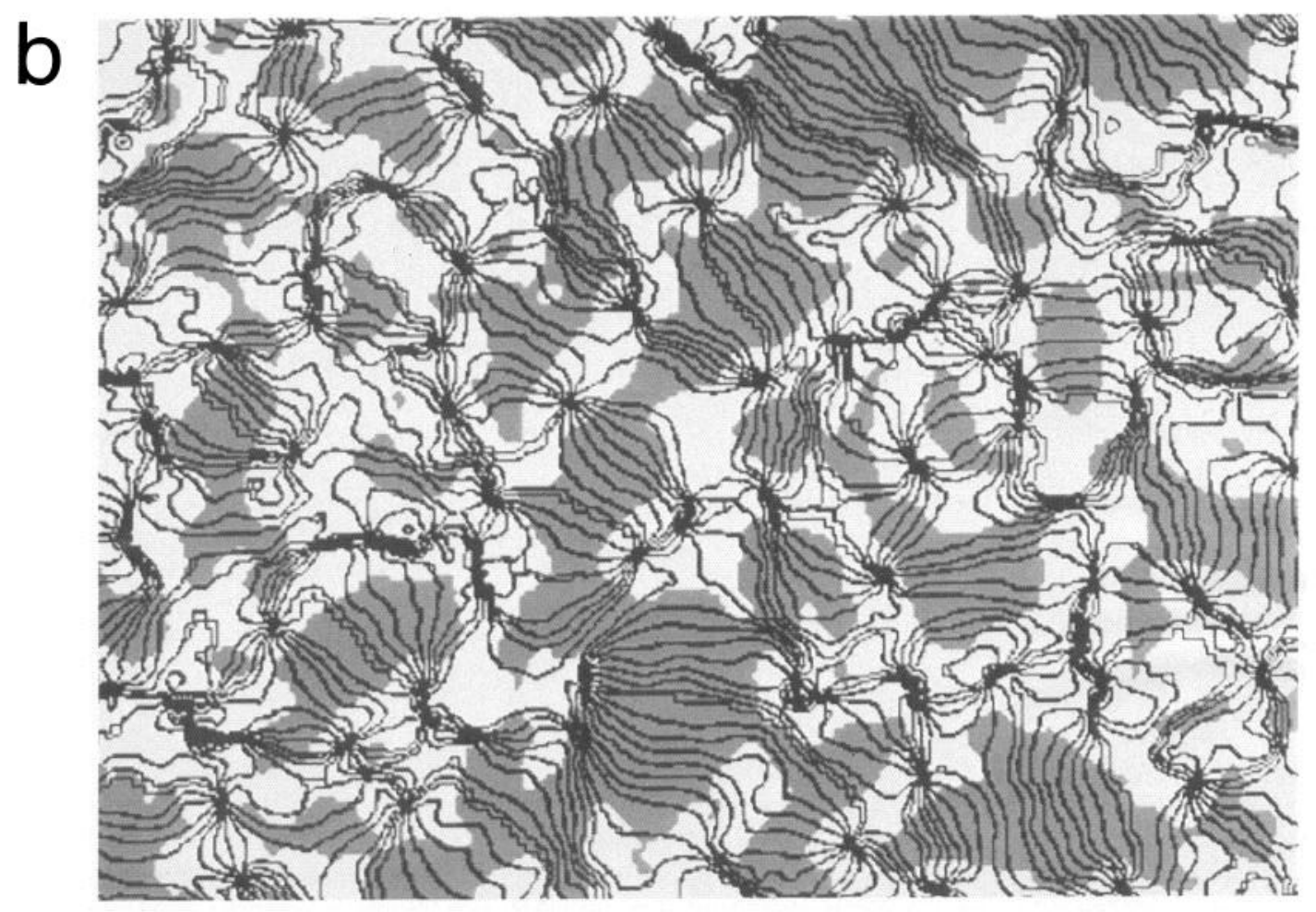

through $90^{\circ}$ (Fig. $6 \mathrm{c}$ ). One might note, moreover, that in the latter case, the inverse differences emerge more weakly because local relationships are not preserved by the rotation (of ocular dominance bands) through $90^{\circ}$. These results are typical of all six areas analyzed, as the histograms in Figure $6 d$ illustrate.

For Figures 1 and 2 it is clear that most of the ocular dom- inance bands run perpendicular to the boundary of striate cortex (the 17/18 border, which runs parallel to the lower border of each frame), as one would expect (LeVay et al., 1975), and that most iso-orientation bands run parallel. Hence, there is a possibility that the observed orthogonality between ocular dominance and orientation is incidental to alignments with the 


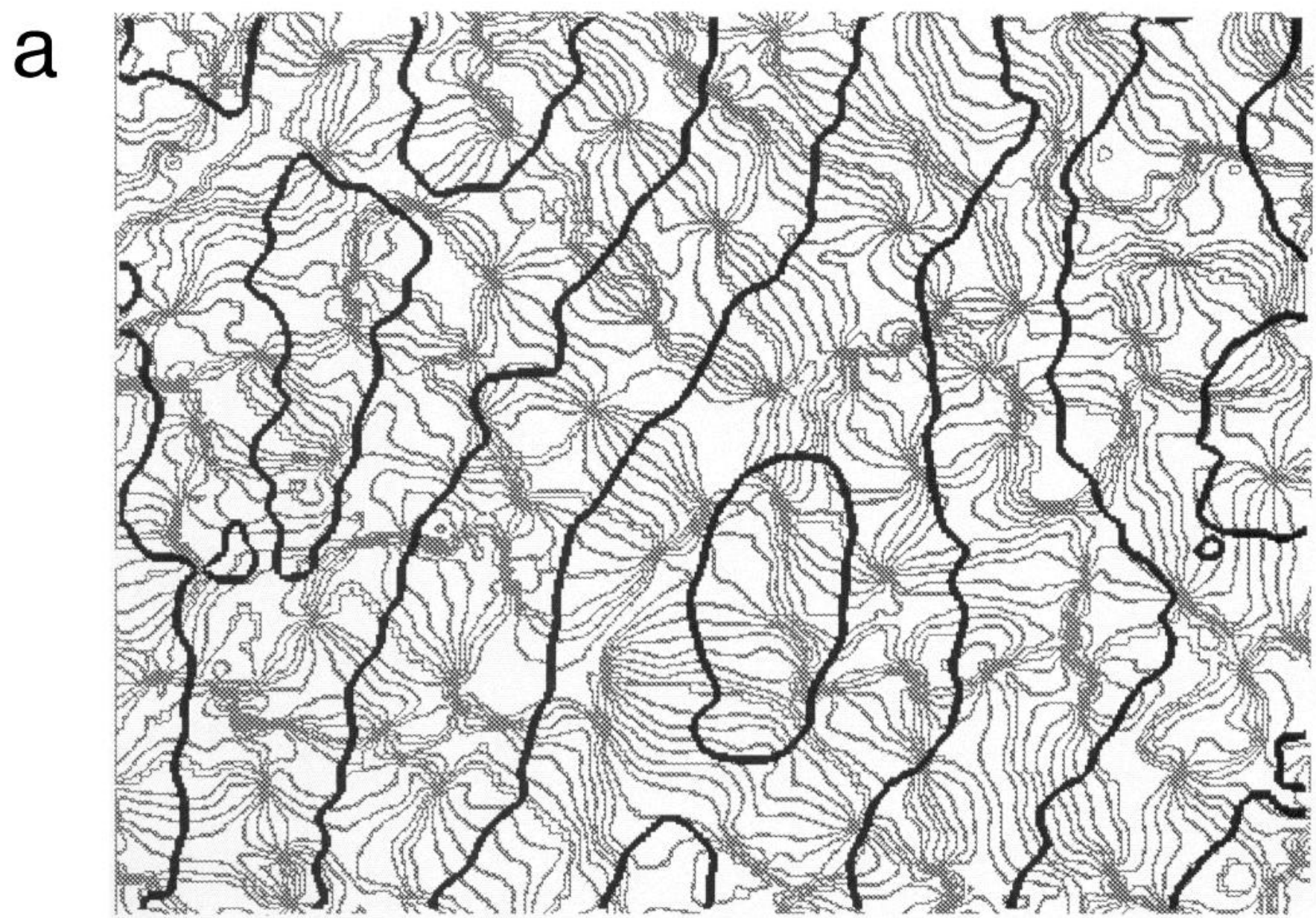

b
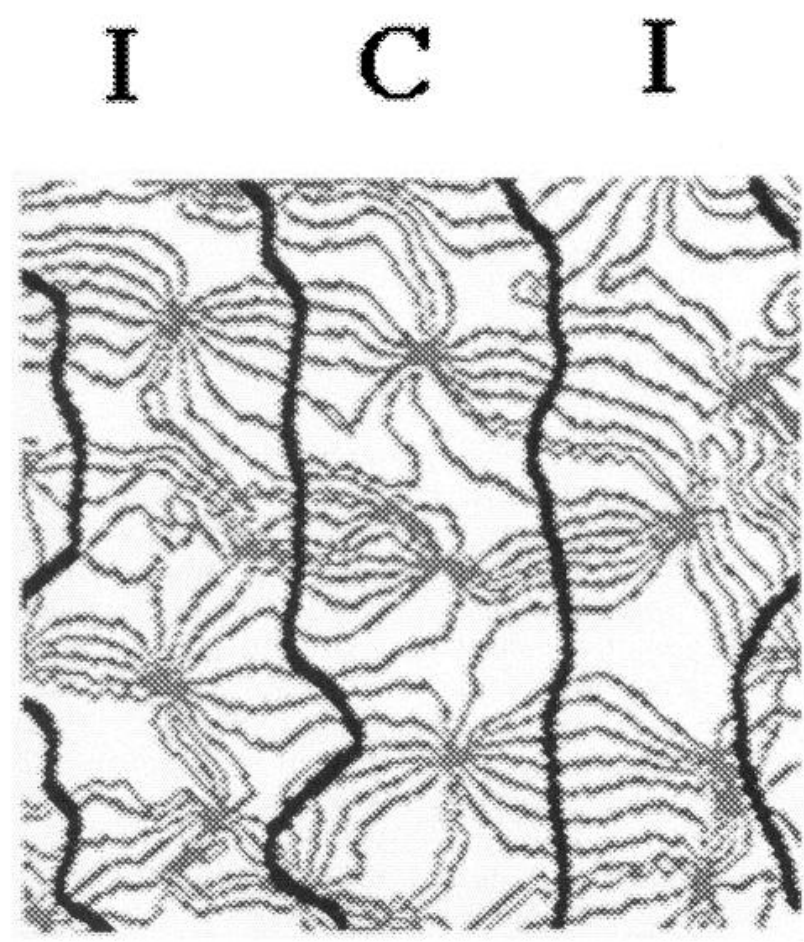

Figure 5. Contour plot of orientation preferences in overlay with the borders of ocular dominance bands. $a$, Complete pattern for animal NM1. Iso-orientation lines (gray) are drawn in intervals of $11.25^{\circ}$. Black lines indicate the border of ocular dominance bands, that is, the locations where the ocular dominance difference signal vanishes. $b$, Enlarged section of the complete pattern. The horizontal axis corresponds to $1.6 \mathrm{~mm}$. $I$ and $C$ denote bands with ipsilateral and contralateral eye preference, respectively. 
a)

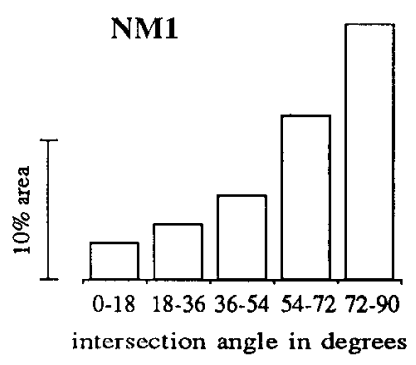

original data b)

NM1

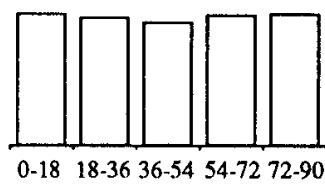

intersection angle in degrees

randomized OD-values c)

NM1

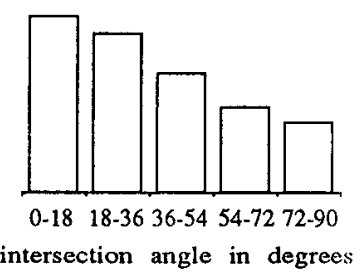

OD-columns rotated by $90^{\circ}$

d)

NM2

Figure 6. Histogram of angles of intersection between orientation and ocular dominance bands. The angle of intersection was calculated for regions characterized by $P O P>0.6$ according to the procedure described in Materials and Methods. $\sigma_{g}$ was $100 \mu \mathrm{m}$. $a$, Percentage of the area of image NM1 that is characterized by a certain angle of intersection. $b$, Percentages of area for NM1 after ocular dominance values have been exchanged between pixels for $5 \times 10^{5}$ randomly selected pairs. $c$, Percentage of the area for NM1 after the ocular dominance pattern has been rotated by $90^{\circ} . d$, Percentage of the area of the images NM2, NM3, NM4, FS11 , and FS1-2, which is characterized by a certain angle of intersection between orientation and ocular dominance bands.

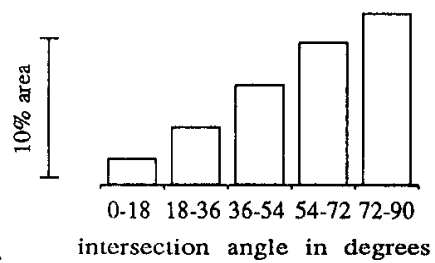

FS1-1

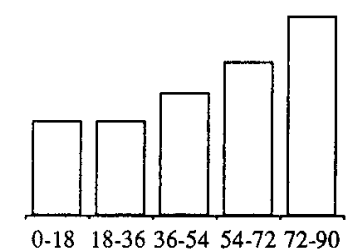

intersection angle in degrees
NM3

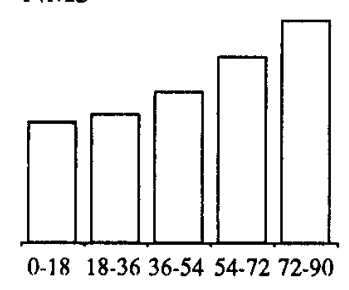

intersection angle in degrees
NM4

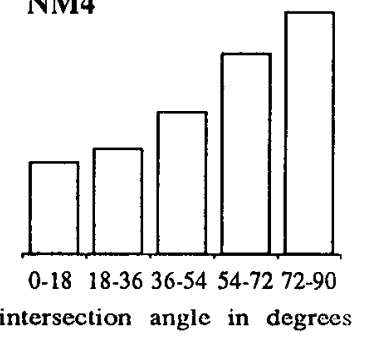

FS1-2

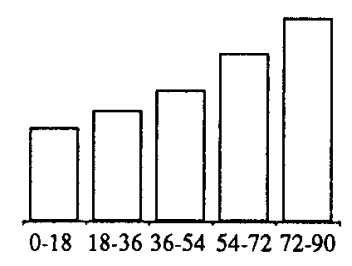

intersection angle in degrees boundaries of striate cortex. This possibility is explored further in Figure 7, where the local orientation of ocular dominance bands is plotted against the local orientation of iso-orientation bands for cortical regions where our measure of linearity exceeds 0.8 . The fact that a large number of data points cluster around one locus $(-0.7,1.1)$ indicates a bias in the angles at which both sets of bands are oriented. This could reflect a general alignment of orientation as well as ocular dominance bands with the $17 / 18$ border (the boundary of striate cortex). However, the fact that many of the data points distribute along a diagonal reflects a tendency for iso-orientation bands to follow changes in the direction of ocular dominance bands locally. This implies that any alignment between iso-orientation bands and the $17 / 18$ border (or between ocular dominance bands and the $17 / 18$ border, for that matter) may occur secondarily to a more fundamental alignment between slabs of iso-orientation and ocular dominance.

The combined histograms of intersection angles for all animals (NM1-NM4, FS1-1, and FS1-2) appear in Figure 8, for regions displaying different values of $P$ P. As one can see, there is a clear correlation between parallelness, as measured by $\mu^{O P}$, and the degree of orthogonality between slabs of ocular dominance and iso-orientation. For regions wherc $P^{O P}>0.8$, for example, ocular dominance and orientation slabs intersect at angles within $18^{\circ}$ of perpendicular $35 \%$ of the time, as compared to $9 \%$ of the time for all values of $P^{\text {OP }}$. The smaller the value of $P^{\mathrm{OP}}$, in fact, the less pronounced the orthogonality. But, even for regions in the immediate vicinity of singularities $\left(0.2<P^{\text {OP }}\right.$ $<0.4$ ), where parallel iso-orientation bands are no longer defined, there is a significant tendency for iso-orientation contours to run perpendicular to the ocular dominance columns, at angles exceeding $45^{\circ}$.

\section{Evaluation of potential artifacts}

\section{Band-pass filtering and postprocessing}

As described in Materials and Methods, the differential images acquired are post processed in a way that approximates the application of a band-pass filter. By filtering out contributions 
from spatial frequencies above and below those of interest, it is possible to improve signal-to-noise ratios appreciably. Highpass filtering is necessary to remove variations in responsiveness and the neutral point between differential images that are to be compared, and low-pass filtering is needed to remove spurious noise. The resulting improvements in signal-to-noise ratio improve the correlations that can be achieved with single-unit recordings at individual test locations (Blasdel and Salama, 1986; G. G. Blasdel, unpublished observations). As with any filtering there are drawbacks, however, that need to be considered.

Where the rate of change of orientation preferences is both high and nonlinear, the local averaging used to improve the signal-to-noise ratio could introduce artifacts that make determinations of orientation selectivity and preference unreliable in the immediate vicinity of fractures and singularities. These artifacts should not affect conclusions in this article, however, since the orthogonality of orientation and ocular dominance slabs is most pronounced in regions where orientation preferences and ocular dominance change smoothly, making their estimates most reliable. Similarly, the classification of local patterns into linear zones, saddle points, and fractures, as well as their approximate localization, should not be compromised either since linear zones and saddle points are not affected by averaging artifacts, and singularities and fractures are global phenomena, which can be inferred from surrounding trends where changes in orientation preference are smooth (Blasdel, 1992b).

Another concern might be that band-pass filters interfere with reliable estimates of characteristic wavelengths and coherence lengths. In the pertinent cases, however, filtering parameters were chosen carefully, such that the amplitudes of the Fourier modes, with wavelengths between $200 \mu \mathrm{m}$ and $2.0 \mathrm{~mm}$ for the high-power images and between $400 \mu \mathrm{m}$ and $2.0 \mathrm{~mm}$ for the low power images, are preserved within $10 \%$ of their value, while estimates of the power are preserved within $20 \%$.

A quantitative evaluation of power spectra (Obermayer et al., $1991,1992 a, b)$ reveals that the power at the border of these intervals is at most $20-30 \%$ of the peak power. Given an attenuation of $20 \%$, the difference in power between the center and the borders of the "reliability interval" is at least $36 \%$. Hence, peaks in the Fourier and power spectra of orientation preference and ocular dominance are pronounced enough to allow estimates of characteristic wavelengths and frequencies within the error margins given. As a consequence, characteristic wavelengths and frequencies can also be calculated directly from the raw data, before postprocessing is done. These have been performed for samples NM1 and NM2, with results matching those displayed in Table 1. However, estimates of the coherence length are disturbed more strongly by these procedures, which affect them by as much as $\pm 16 \%$.

\section{Vascular artifacts}

As anyone who has ever studied neocortex can appreciate, striate cortex is covered by a thick mat of blood vessels that are very difficult to avoid. Most of these are small and, at the wavelengths used, transparent (Blasdel, 1992a,b). There are instances where vascular artifacts intrude nevertheless, and need to be considered. For the analyses performed in this article, most of the obviously affected regions have been removed (see hatching, Fig. 1). It is important to point out, however, that even where we may have failed to eliminate all traces of vascular artifact, they are not likely to produce these results since there are no

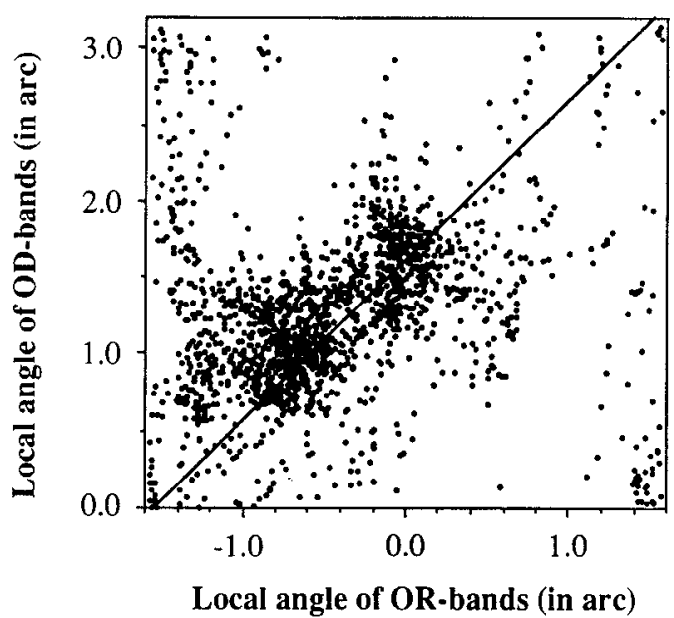

Figure 7. Graph showing the local oricntation of ocular dominance bands as a function of the local orientation of iso-orientation slabs for regions characterized by $P$ OP $>0.8$ for animal NM1. The directions of iso-orientation and ocular dominance bands were calculated every 17.2 $\mu \mathrm{m}$, that is, at every second pixel location. $\sigma_{g}$ was $100 \mu \mathrm{m}$.

known correlations between the vasculature and ocular dominance (Blasdel, 1992a), or between the vasculature and orientation selectivity (Blasdel, 1992b). Hence, any vascular artifacts should either randomize the data (if they affect only one set of images) or bring them into alignment (if they affect both). In either case it would be difficult for such artifacts to make isoorientation slabs appear to intersect ocular dominance borders at right angles. In this regard it is also important to note that we observe the strongest correlations in areas where the probability of vascular artifacts is least (e.g., NMl).

\section{Border effects}

Since many of the optical images in this article were obtained from cortical areas close to the $17 / 18$ border, where ocular dominance columns are known to be more aligned and to intersect the boundary of striate cortex at right angles (LeVay et al., 1975), there is a possibility that these correlations are not representative of other parts of the visual field. While the optical imaging strategy employed here is limited to regions of cortex that are accessible, the possible significance of border proximity is reduced by the facts (1) that the strongest correlations were obtained for a region of cortex located more than $1 \mathrm{~cm}$ away (NM1), and (2) that in the animal (FS1) where patterns were derived from two scparate arcas, the corrclations are not any stronger in the area $3 \mathrm{~mm}$ from the $17 / 18$ border (FS1-1) than they are in the area $9 \mathrm{~mm}$ away (FS1-2). The regularity of ocular dominance bands also appears not to be a factor in these correlations since the apparent orthogonality between ocular dominance and orientation is just as pronounced in the two regions imaged from FS1, where ocular dominance bands are noticeably irregular, as they are in other animals.

\section{Discussion}

These results confirm and extend previous observations concerning the organization of orientation preference and ocular dominance in monkey striate cortex. In agreement with Hubel and Wiesel (1974a, 1977), they provide quantitative evidence that orientation preferences change linearly with distance, across much of the cortical surface, but they also quantitate subsequent 


\section{original data}
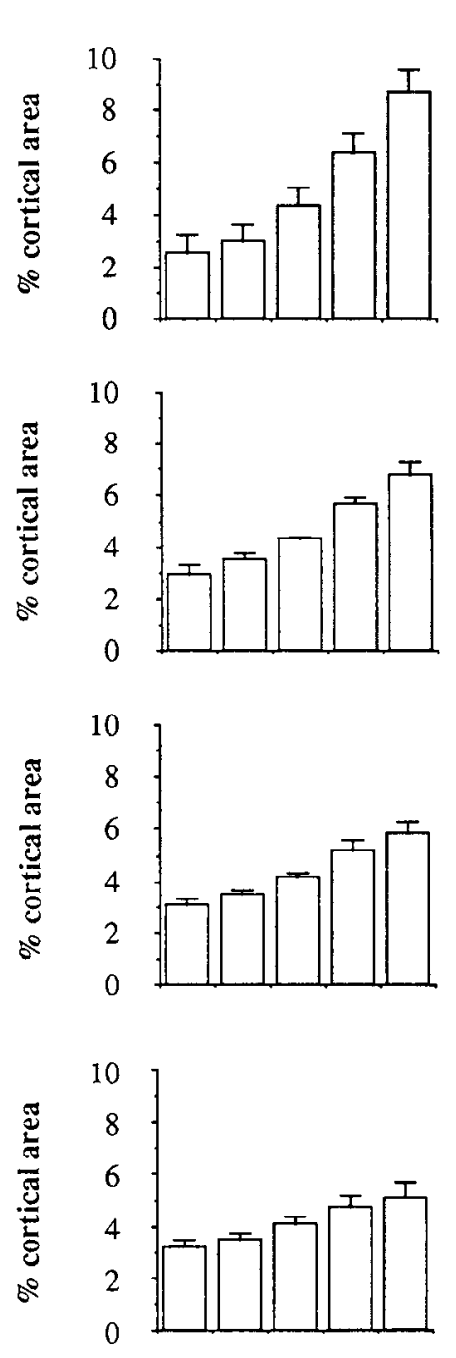

Figure 8. Average histogram of angles of intersection as a function of the measure $P^{\mathrm{OP}}$ of "parallelness." Left column, The percentage of the total area of all images (NM1-NM4, FS1-1, and FS12) is plotted against the local angle of intersection between orientation and ocular dominance bands. Histograms were obtained for different values of $P^{\circ}$. Right column, These histograms show the percentage of the total area after ocular dominance values have been exchanged between pixels for $5 \times 10^{5}$ randomly selected pairs for each map. $\sigma_{g}$ was $100 \mu \mathrm{m}$. All error bars indicate SEM.

$$
\text { 宽 }
$$

\section{randomized OD-values}

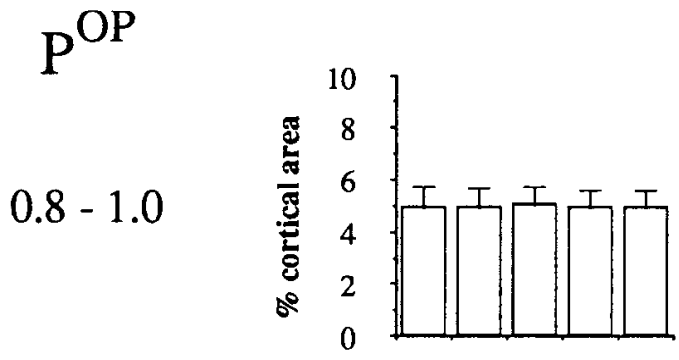

$0.6-0.8$
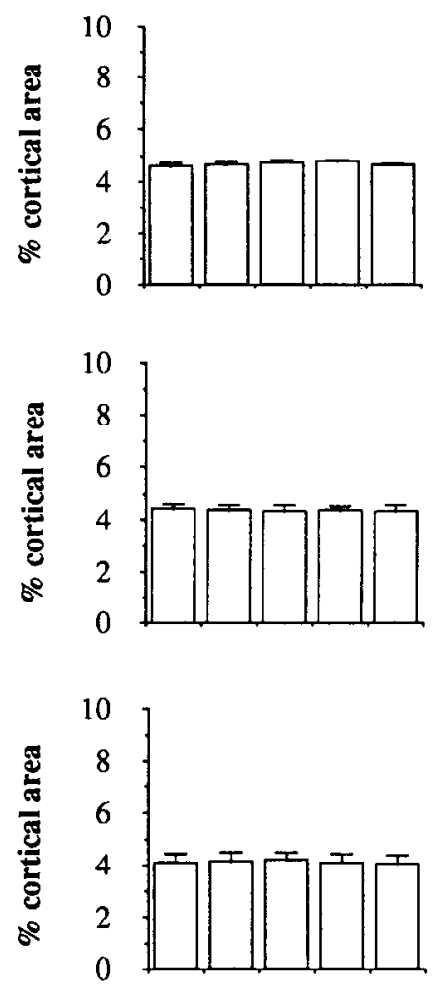

$0.2-0.4$
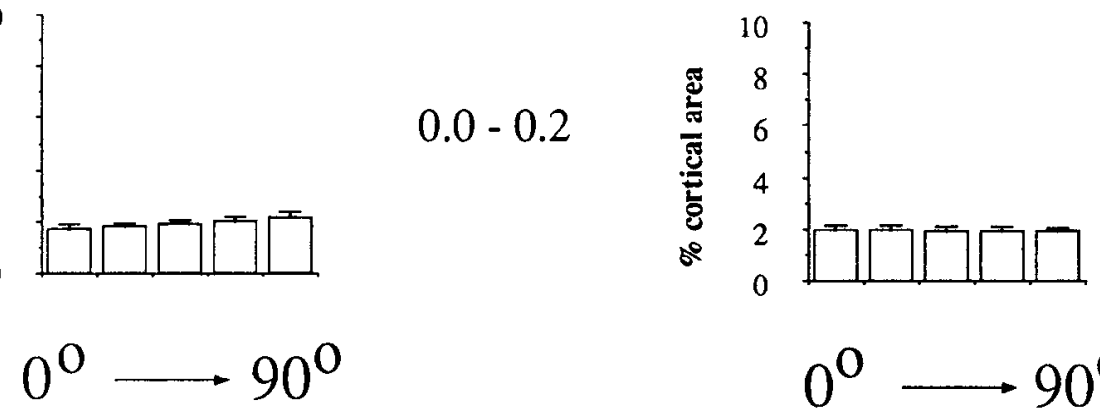

\section{intersection angle in degrees}

observations (Blasdel and Salama, 1986; Blasdel, 1992b) that such changes are confined to patch-like linear zones, 0.5-1.0 $\mathrm{mm}$ across, segregated by nonlinear boundaries. These results also reveal precise geometric relations between patterns of ocular dominance and orientation in three separate ways: (1) they show that orientation preferences repeat at closer intervals along ocular dominance columns than across them, (2) they confirm that linear and nonlinear zones correlate with the edges and centers of ocular dominance columns, and (3) they establish that, in the linear zones, iso-orientation slabs cross ocular dominance edges at essentially perpendicular angles.

\section{Comparison with previous work}

By far the most intriguing finding is that bands of iso-orientation and ocular dominance intersect at nearly perpendicular angles. This possibility was suggested by Hubel and Wiesel (1974a), initially, for theoretical reasons that are compelling (see below). When it was tested explicitly, however, it appeared not to be 
true. After giving $2 \mathrm{DG}$ to animals stimulated with a single orientation, Hubel et al. (1978) observed that the bands of label harvested in striate cortex intersected ocular dominance boundaries at all angles. Blasdel and Salama (1986) performed a similar experiment, by comparing band trajectories in differential images of orientation and ocular dominance, and obtained a similar result; the bands of orientation and ocular dominance that seemed apparent did not cross at any consistent angle. Upon further reflection it became clear, however, that single 2DG autoradiograms and single differential images are ambiguous with respect to orientation, for it is only by comparing responses to many orientations that preferences become defined (Blasdel, 1992a). This point becomes particularly clear when the bands apparent in single differential images are compared with actual iso-orientation bands determined from responses to many different orientations (see Fig. 19 in Blasdel, 1992a, for example). In spite of considerable overlap, most iso-orientation bands follow independent trajectories that cannot be inferred from any single differential image.

When orientation preferences are calculated precisely, however, from several differential images, correlations with ocular dominance become apparent. As Blasdel and Salama (1986) reported initially, the nonlinear borders (e.g., fractures, singularities) between linear zones are highly concentrated in the centers of ocular dominance columns. It then was noticed (Obermayer et al., 1992a,b; Blasdel, 1992b) that short slabs of isoorientation within the linear zones cross the borders between ocular dominance columns at steep angles.

\section{Local architectures}

In addition to providing quantitative evidence for the geometrical relationship between ocular dominance and orientation slabs, these studies provide quantitative descriptions of four patterns of orientation preference that may be discerned locally.

Linear zones. By far the most common of these are the twodimensional linear zones, where orientation preferences rotate linearly along one straight axis while remaining constant along the other. These zones are also recognizable as the stacks of parallel orientation slabs deduced by Hubel and Wiesel (1974a). These zones are two dimensional, extending $0.5-1.0 \mathrm{~mm}$ across, and given their relative abundance in striate cortex, it is little wonder that they played such a prominent role in Hubel and Wiesel's (1974a) initial studies of the orientations encountered during long tangential penetrations through monkey striate cortex. The patch-like dimensions of thesc linear zoncs werc apparent in the first optical maps of orientation preference (Blasdel and Salama, 1986), and arguably could have been predicted even earlier, on the basis of Hubel and Wiesel's (1974a) findings. Even though their linearity has recently been questioned (Bonhoeffer and Grinvald, 1991; Bartfeld and Grinvald, 1992), these results provide strong quantitative evidence for them.

By calculating the aligmment of iso-orientation contours in discrete regions, $200 \mu \mathrm{m}$ across, one can estimate the degree of linearity from local factors as well. When this is done for all six regions, depicted in Figure 1, it becomes clear that half the cortical surface is characterized by alignment values in excess of 0.6. A linear regression analysis performed in these regions leads to a correlation coefficient of 0.994 , which shows that regions of alignment are indeed characterized by linear changes of orientation preference. A detailed analysis of the Fourier spectra in Figure 2, moreover, revealed a coherence length of approximately $820 \mu \mathrm{m}$, which indicates the approximate size of the linear zones and which corresponds to the dimension estimate of $0.5-1.0 \mathrm{~mm}$, derived from casual inspection (Blasdel, 1992b).

Exactly what the linear zones do remains to be clarified. They are characterized by linear maps for orientation running perpendicular to linear maps for ocular dominance (and/or retinotopic location), in the most binocular regions of cortex. By analogy with Hough transforms (Hough, 1962; Ballard et al., 1983), therefore, it is conceivable that cells in these zones are interested in boundary contours (Grossberg, 1985; Blasdel, 1992b).

Singularities. These were predicted by Braitenberg and Braitenberg (1979), Swindale (1992), Durbin and Mitchison (1990), and Obermayer et al. (1990) on the basis of mapping considerations. They were observed in the first optical maps of orientation from monkey striate cortex (Blasdel and Salama, 1986; Götz, 1987; Blasdel, 1989b, 1992b), and were reported in cat area 18 by Swindale et al. (1987) on the basis of single-unit studies, as well as by Bonhoeffer and Grinvald (1991), on the basis of optical recordings.

The fact that orientation preferences rotate through exactly $\pm 180^{\circ}$, in one revolution about each singularity, gives them interesting properties, for this guarantees that orthogonal orientations are represented as close together as possible, on opposite sides. The fact that upper layer pyramidal cell dendrites fill cylindrical regions, 200-300 $\mu \mathrm{m}$ across (Lund and Yoshioka, 1991), raises the intriguing possibility that cells near singularities might be uniquely positioned to compare edge distributions at orthogonal orientations, which raises the additional possibility that they are concerned with texture discrimination (Blasdel, 1992b).

Fractures. During their long tangential recordings through the upper layers of monkey striate cortex, Hubel and Wiesel (1974a) observed (and verified) sudden, abrupt shifts in orientation preference, through angles as large as $45^{\circ}$ and at intervals of $0.5-1.5 \mathrm{~mm}$. The subsequent recordings of Blasdel and Salama (1986) revealed a likely suurce for these shifts, in the form of fractures, or lines of discontinuous change, that run between adjacent linear zones. Fractures are apparent as closely spaced lines in the isocontour plots of Figures 3 and 5, and, while it could be argued that these closely spaced lines represent "elongated zones of more rapid change," this is at best a semantic distinction since the linearity characterizing adjacent regions is disrupted in any case.

Since they see singularities more easily than fractures, Bonhoeffer and Grinvald (1991) and Bartfeld and Grinvald (1992) have suggested that orientation preferences in visual cortex are organized in pinwheels, around singularities, rather than in slabs in linear zones. This formulation was originally made by Braitenberg and Braitenberg (1979), and is not too different from ours since linear zones can be discerned in pinwheel architectures as well. The difficulty that Bonhoeffer and Grinvald (1991) and Bartfeld and Grinvald (1992) have had in resolving fractures, however, which can be seen with microelectrodes (Hubel and Wiesel, 1974a), suggests a limited spatial resolution to their technique that might arise from a greater depth of signal integration in the absence of light-absorbing dye (Blasdel, 1992b). This is particularly likely in the studies of cat visual cortex (Bonhoeffer and Grinvald, 1991), where the pronounced curvature of the lateral gyrus makes it likely that borders of sharp change are blurred.

Saddle points. Thesc arc apparent in all previously published maps of orientation preference from monkey striate cortex, but are singled out as entities for the first time. They consist of 
continuous two-dimensional distributions, where the gradient of orientation preferences reverses direction. As one traverses the center of a saddle point, therefore, the direction of change (e.g., clockwise or counterclockwise) inverts direction. For outward displacements (in opposite directions) from the center, on the other hand, orientation preferences rotate in similar directions. Saddle points are usually bounded by four singularities that may, or may not, be linked by fractures. In all probability the saddle points we observe correspond to the regions where Hubel and Wiesel (1974a) observed the linear changes in orientation preference to reverse direction.

\section{Orthogonality between orientation preference and ocular dominance}

The observed orthogonality between ocular dominance and orientation could derive from several considerations. As Hubel and Wiesel argued initially (1974a, 1977), mechanisms associated with ocular dominance and orientation may engage in separate perceptual functions. The fact that orientation and ocular dominance slabs extend across overlapping regions of cortex, therefore, and that they additionally are characterized by similar periods of repetition, suggests a need for mechanisms to ensure that these response properties remain independent (and uncorrelated). Orthogonal trajectories for orientation and ocular dominance slabs could achieve this, even if the periods of repetition are the same.

The orthogonality between orientation and ocular dominance could also derive from coverage constraints governing the need to represent all relevant combinations of orientation preference and ocular dominance in all parts of visual space. If coverage is incomplete, for example, there could be perceptual scotomata in certain parts of the visual field. It is interesting to note in this regard that if ocular dominance and orientation are both organized in slabs, with approximately equal periods, then only an orthogonal arrangement can optimize coverage (Swindale, 1991).

Another possibility is that the orthogonality between ocular dominance and orientation relates to an anisotropic mapping of the visual field. As Hubel et al. (1978) found initially, and Blasdel and Fitzpatrick (1984) subsequently explored in greater detail, the ocular dominance bands in layer $4 \mathrm{c}$ represent the visual field twice-once for either eye-in directions perpendicular to the ocular dominance columns, such that the representation of points that appear equally spaced in space lie farther apart across the ocular dominance columns than they do along them. This anisotropic spread also characterizes the distribution of lateral connections (Blasdel et al., 1992; Yoshioka et al., 1992). Accordingly, it would not be surprising to find that the longer repetition periods of orientation preferences perpendicular to the ocular dominance columns (detailed in the Fourier transforms in Fig. 2) derive from anisotropic mapping as well. In this case the elongated, slab-like appearance of iso-orientation columns might arise from little more than a stretched, anisotropic representation of visual space.

It is also possible to argue the converse, however, that striate perceptual operations require linear maps of orientation preference, which then induce ocular dominance columns to segregate in the perpendicular direction, perhaps because they need balanced input from both eyes, for the same parts of visual space (Blasdel, 1992b). This possibility, that parallel iso-orientation slabs may be the cause, rather than the consequence, of ocular dominance columns is furthermore consistent with the available developmental evidence suggesting that linear maps of orientation appear long beforc the scgregated bands of afferents in layer 4c (Wiesel and Hubel, 1974; LeVay et al., 1980).

\section{References}

Ballard D, Hinton J, Sejnowski T (1983) Parallel visual computation. Nature 306:21-26.

Bartfeld E, Grinvald A (1992) Relationships between orientation-preference pinwheels, cytochrome oxidase blobs, and ocular-dominance columns in primate striate cortex. Proc Natl Acad Sci USA 89:1190511909.

Blasdel GG (1989a) Topography of visual function as shown with voltage sensitive dyes. In: Sensory systems in the mammalian brain (Lund JS, ed), pp 242-268. New York: Oxford UP.

Blasdel GG (1989b) Visualization of neuronal activity in monkey striate cortex. Annu Rev Physiol 51:561-581.

Blasdel GG (1992a) Differential imaging of ocular dominance and orientation selectivity in monkey striate cortex. J Neurosci 12:31173138.

Blasdcl GG (1992b) Oricntation sclectivity, preferencc, and continuity in monkey striate cortex. J Neurosci 12:3139-3161.

Blasdel GG, Fitzpatrick D (1984) Physiological organization of layer 4 in macaque striate cortex. J Neurosci 4:880-895.

Blasdel GG, Salama G (1986) Voltage sensitive dyes reveal a modular organization in monkey striate cortex. Nature 321:579-585.

Blasdel GG, Yoshioka T, Levitt JB, Lund JS (1992) Correlation between patterns of lateral connectivity and patterns of orientation preference in monkey striate cortex. Soc Neurosci Abstr 18:389.

Bonhoeffer T, Grinvald A (1991) Orientation columns in cat are organized in pinwheel like patterns. Nature 353:429-431.

Braitenberg V, Braitenberg C (1979) Geometry of orientation columns in the striate cortex. Biol Cybern 33:179-186.

Durbin R, Mitchison G (1990) A dimension reduction framework for understanding cortical maps. Nature 343:644-647.

Frostig RD, Lieke EE, Ts'o DY, Grinvald A (1990) Cortical functional architecture and local coupling between neuronal activity and the microcirculation revealed by in vivo high-resolution optical imaging of intrinsic signals. Proc Natl Acad Sci USA 87:6082-6086.

Götz KG (1987) Do "d-blob" and "l-blob" hypercolumns tesselate the monkey visual cortex? Biol Cybern 56:107-109.

Horton JC, Hubel DH (1980) Regular patchy distribution of cytochrome oxidase staining in primary visual cortex of the macaque. Nature 292:762-764.

Hough PVC (1962) Method and means for recognizing complex patterns. US Patent \#3069654.

Hubel DH, Wiesel T (1962) Receptive fields, binocular interaction, and functional architecture of monkey striate cortex. J Physiol (Lond) 160:106-154.

Hubel DH, Wiesel T (1968) Receptive fields and functional architecture of monkey striate cortex. J Physiol (Lond) 195:215-243.

Hubel DH, Wiesel TN (1972) Laminar and columnar distribution of geniculo-cortical fibers in the macaque monkey. J Comp Neurol $146: 421-450$.

Hubel DH, Wiesel TN (1974a) Sequence regularity and geometry of orientation columns in the monkey striate cortex. J Comp Neurol 158:267-294.

Hubel DH, Wiesel TN (1974b) Uniformity of monkey striate cortex: a parallel relationship between field size, scatter, and magnification factor. J Comp Neurol 158:295-306.

Hubel DH, Wiesel TN (1977) Functional architecture of macaque monkey visual cortex. Proc R Soc Lond [Biol] 198:1-59.

Hubel DH, Wiesel TN, Stryker MP (1978) Anatomical demonstration of orientation columns in macaque monkey. J Comp Neurol 177: 361-380.

Humphrey AL, Hendrickson AE (1983) Background and stimulusinduced patterns of high metabolic activity in the visual cortex (area 17) of the squirrel and the macaque monkey. J Neurosci 3:345-358.

LeVay S, Hubel DH, Wiesel TN (1975) The pattern of ocular dominance columns in macaque striate cortex revealed by a reduced silver stain. J Comp Neurol 159:559-576.

LeVay S, Wiesel TN, Hubel DH (1980) The development of ocular 
dominance columns in normal and visually deprived monkeys. J Comp Neurol 191:1-51.

Livingstone MS, Hubel DH (1982) Thalamic inputs to cytochrome oxidase-rich regions in monkey visual cortex. Proc Natl Acad Sci USA 79:6098-6101.

Livingstone MS, Hubel D (1984) Anatomy and physiology of a color system in the primary visual cortex. J Neurosci 4:309-356.

Lund JS, Yoshioka T (1991) Local circuit neurons of macaque monkey striate cortex III. Neurons of laminae 4B, 4A and 3B. J Comp Neurol 311:234-258.

Obermayer K, Ritter H, Schulten K (1990) A principle for the formation of cortical feature maps. Proc Natl Acad Sci USA 87:83458349 .

Obermayer K, Blasdel GG, Schulten K (1991) A neural network model for the formation and for the spatial structure of retinotopic maps, orientation- and ocular dominance columns. In: Artificial neural networks (Kohonen T et al,, eds), pp 505-511. Amsterdam: ElsevierNorth Holland.

Obermayer K, Blasdel GG, Schulten K (1992a) A statistical mechanical analysis of self-organization and pattern formation during the development of visual maps. Phys Rev [A] 45:7568-7589.

Obermayer K, Schulten K, Blasdel GG (1992b) A comparison of a neural network model for the formation of brain maps with experimental data. In: Advance in neural information processing systems (Touretzky DS, Lippman R, eds), pp 83-90. San Mateo, CA: Morgan Kaufmann.
Papoulis A (1984) Probability, random variables, and stochastic processes. New York: McGraw-Hill.

Press WH, Flannery BP, Teukolsky SA, Vetterling WT (1988) Numerical recipes in C. Cambridge: Cambridge UP.

Swindale NV (1991) Coverage and the design of striate cortex. Biol Cybern 65:415-424.

Swindale NV (1992) A model for the coordinated development of columnar systems in primate striate cortex. Biol Cybern 66:217-230.

Swindale NV, Matsubara JA, Cynader MS (1987) Surface organization and direction selectivity in cat area 18 . J Neurosci 7:1414-1427.

Tootell RBH, Switkes E, Silverman MS, Hamilton SL (1988) Functional anatomy of macaque striate cortex. II. Retinotopic organization. J Neurosci 8:1531-1568.

Ts'o DY, Frostig RD, Lieke EE, Grinvald A (1990) Functional organization of primate visual cortex revealed by high resolution optical imaging. Science 249:417-420.

Wiesel TN, Hubel DH (1974) Ordered arrangement of orientation columns in monkeys lacking visual experience. J Comp Neurol 158: 307-318.

Wong-Riley MTT (1979) Changes in the visual system of monocularly sutured or enucleated cats demonstrable with cytochrome oxidase histochemistry. Brain Res 171:11-28.

Yoshioka T, Blasdel GG, Levitt JB, Lund JS (1992) Patterns of lateral connections in macaque visual area $\mathrm{V} 1$ revealed by biocytin histochemistry and functional imaging. Soc Neurosci Abstr 18:299. 